\title{
TREINAMENTO DE HABILIDADES FONOLÓGICAS E CORRESPONDÊNCIA GRAFEMA-FONEMA EM CRIANÇAS DE RISCO PARA DISLEXIA
}

\begin{abstract}
Phonological skills and grapheme-phoneme training correspondence in children under dyslexia risk
\end{abstract}
\author{
Maryse Tomoko Matsuzawa Fukuda ${ }^{(1)}$, Simone Aparecida Capellini ${ }^{(2)}$
}

\begin{abstract}
RESUMO
Objetivo: verificar a eficácia do programa de treinamento fonológico e correspondência grafemafonema em crianças de risco para dislexia da 1a série. Método: participaram deste estudo 30 crianças de $1^{\text {a }}$ série de ensino público, de ambos os gêneros, na faixa etária de 6 a 7 anos de idade. Neste estudo foi realizada a adaptação brasileira da pesquisa sobre treinamento de habilidades fonológicas e correspondência grafema-fonema composta de pré-testagem, intervenção e pós-testagem. Em situação de pré e pós-testagem, todas as crianças foram submetidas à aplicação do teste para identificação precoce dos problemas de leitura e aquelas que apresentaram desempenho inferior a $51 \%$ das provas do teste foram divididas em Grupo I (Gl): composto por 15 crianças submetidas ao programa de treinamento; e em Grupo II (GII): composto por 15 crianças não submetidas ao programa de treinamento. Resultados: os resultados desse estudo revelaram diferenças estatisticamente significantes, evidenciando que das 15 crianças submetidas ao programa, apenas uma não respondeu à intervenção proposta, sendo submetida, portanto, à avaliação interdisciplinar e confirmado o diagnóstico de dislexia. Conclusão: a realização do programa de treinamento das habilidades fonológicas e correspondência grafema-fonema foi eficaz para a identificação das crianças com sinais de dislexia, comprovado pela melhora das habilidades fonológicas e leitura em situação de pós-testagem em relação à pré-testagem, evidenciando que quando é fornecida a instrução formal do princípio alfabético associado ao principio de conversão letra-som da Língua Portuguesa, as crianças que não apresentam dislexia deixam de apresentar suas manifestações com resposta à instrução formal do princípio alfabético.
\end{abstract}

DESCRITORES: Dislexia; Avaliação; Intervenção Precoce (Educação); Leitura; Criança

\section{INTRODUÇÃO}

Dislexia é um distúrbio específico de aprendizagem de origem neurológica; caracteriza-se pela dificuldade com a fluência correta na leitura e dificuldade na habilidade de decodificação e soletração,

(1) Fonoaudióloga Voluntária do Departamento de Fonoaudiologia da Faculdade de Filosofia e Ciências da Universidade Estadual Paulista, FFC/UNESP, Marília, SP, Brasil.

(2) Fonoaudióloga; Professora do Departamento de Fonoaudiologia e Programa de Pós-Graduação em Educação da Faculdade de Filosofia e Ciências da Universidade Estadual Paulista, FFC/UNESP, Marília, SP, Brasil; Doutora e Pós-Doutora em Ciências Médicas pela Faculdade de Ciências Médicas da Universidade Estadual de Campinas.

Conflito de interesses: inexistente resultantes de um déficit no componente fonológico da linguagem ${ }^{1}$.

Os principais sinais da dislexia podem ser evidenciados durante o desenvolvimento da criança ${ }^{2}$, a saber: fala ininteligível; imaturidade fonológica; redução de léxico; dificuldade em aprender o nome das letras ou os sons do alfabeto; dificuldade para entender instruções, compreender a fala ou material lido; dificuldade para lembrar números, letras em sequência, questões e direções; dificuldade para lembrar sentenças ou estórias; atraso de fala; confusão direita-esquerda, embaixo, em cima, frente-atrás (palavras-conceitos) e dificuldade em processar os sons das palavras.

Como estes sinais podem ser evidenciados em fase pré-escolar e em início da alfabetização, 
a dislexia pode ser identificada e detectada precocemente. Estudos internacionais recomendam o uso de intervenção em crianças de séries iniciais de alfabetização que apresentam os sinais da dislexia ${ }^{3-5}$.

Segundo estes estudos, o objetivo desta intervenção é verificar se após a realização de programas específicos com as habilidades cognitivo-linguísticas alteradas, as crianças apresentam melhora na aprendizagem da leitura ou se permanecem com as defasagens nessas habilidades, o que significa que as crianças realmente apresentam uma desordem de origem genético-neurológica que compromete a aquisição e o desenvolvimento de habilidades perceptivas e linguísticas e que por isso deveriam ser submetidas a avaliações interdisciplinares para a investigação do quadro de dislexia.

A importância para a prática clínica e educacional destes estudos encontra-se na ênfase na falta de resposta à intervenção precoce com os sinais da dislexia, que pode ser considerada um critério para o diagnóstico da dislexia do desenvolvimento ${ }^{6}$.

Um estudo realizado com 57 crianças préescolares não leitoras teve por objetivo analisar as alterações na habilidade de conhecimento de letras no início de um programa de intervenção e, depois de cinco meses, determinar como o desenvolvimento desta habilidade se modificou ao longo deste período e como se relacionou com o processamento fonológico e a detecção de rima. Os resultados evidenciaram ganhos estatisticamente significantes para a identificação de letras quando relacionadas com a habilidade fonológica e que as crianças que obtiveram pouco ganho significante para o conhecimento de letras apresentaram atraso em relação ao grupo-classe no desempenho das habilidades cognitivas ${ }^{7}$.

$\mathrm{Na}$ literatura ${ }^{8-10}$ há referência de que a consciência fonológica, o conhecimento de letras e a nomeação rápida são importantes preditores para o desenvolvimento da leitura. Por isso, estudos internacionais apontam para a necessidade de realização de intervenção precoce em crianças que apresentam desempenho abaixo do esperado em fase pré-escolar e nos dois primeiros anos de alfabetização em relação ao seu grupo-classe nos fatores preditivos para o bom desempenho em leitura, como: conhecimento do alfabeto, nomeação automática rápida, repetição de não-palavras e habilidades de consciência fonológica. Estas crianças são denominadas na literatura internacional como crianças de risco para a dislexia ${ }^{3,5,6,11-13}$.

Desta forma, é preciso que tanto professores como fonoaudiólogos estejam atentos ao desenvolvimento da linguagem expressiva e receptiva das crianças no início da alfabetização para que a identificação e a detecção dos sinais da dislexia possam ocorrer precocemente e os problemas acadêmicos decorrentes de alterações cognitivo-linguísticas minimizados por meio da realização de programas de treinamento de habilidades fonológicas associado à correspondência grafema-fonema.

Com base no exposto, este estudo tem por objetivo verificar a eficácia do programa de treinamento de habilidades fonológicas e correspondência grafema-fonema em crianças de risco para a dislexia da $1^{\text {a }}$ série.

\section{MÉTODO}

Este estudo se caracteriza por ser experimental e transversal realizado com crianças de ensino público municipal da cidade de Marília-SP.

Participaram deste estudo 30 crianças de $1^{\text {a }}$ série do ensino público fundamental municipal, de ambos os gêneros e na faixa etária entre 6 a 7 anos de idade (Tabela 1).

Tabela 1 - Distribuição das crianças da 1asérie submetidas à avaliação para a identificação dos sinais da dislexia segundo gênero

\begin{tabular}{|c|c|c|}
\hline GRIPOS & MASCULINO & FEMININO \\
\hline Ghurus & N $\quad(\%)$ & N (\%) \\
\hline GI & $10(33 \%)$ & $5(18 \%)$ \\
\hline GII & $5(18 \%)$ & 10 (33\%) \\
\hline
\end{tabular}

As crianças que participaram deste estudo não apresentaram anotações referentes a deficiências mentais, físicas, sensoriais ou múltiplas em prontuário escolar.

A identificação do risco para dislexia nas crianças deste estudo foi realizada a partir da elaboração de provas de avaliação descritas na pesquisa sobre treinamento de habilidades fonológicas e conhecimento de letra-som em crianças de risco para dislexia ${ }^{3}$. Inicialmente todas as crianças deste estudo foram submetidas aos seguintes procedimentos:

- Termo de Consentimento Livre e Esclarecido: conforme resolução do Conselho Nacional de Saúde CNS 196/96, anteriormente ao início do estudo, os pais ou responsáveis pelas crianças selecionadas assinaram o Termo de Consentimento autorizando a aplicação dos procedimentos deste estudo.

- Teste para identificação precoce dos problemas de leitura: Foi realizada a elaboração de sete provas para identificação precoce dos problemas de leitura baseadas na descrição da 
pesquisa original que oferece suporte teórico para a realização deste estudo. As provas que compõem o Teste estão descritas a seguir:

1. Conhecimento do alfabeto: foi apresentado à criança o alfabeto para que esta identificasse o nome da letra e o valor sonoro de cada letra apresentada.

2. Consciência fonológica: composta por subtestes de produção de rima, identificação de rima, segmentação silábica, produção de palavras a partir do fonema dado, síntese fonêmica, análise fonêmica, identificação de som inicial.

2.1. Produção de rima: foram apresentadas à criança 20 palavras auditivamente e solicitado que dissesse uma palavra que terminasse com o mesmo som.

2.2. Identificação de rima: foram apresentados à criança 20 grupos de três em três palavras auditivamente e solicitado que identificasse as palavras que terminassem com o mesmo som.

2.3. Segmentação silábica: foram apresentadas à criança 21 palavras auditivamente (dissílabas, trissílabas e quadrissílabas) e solicitado que separasse as palavras por sílabas.

2.4. Produção de palavras a partir do fonema dado: foram apresentados à criança os sons do alfabeto e solicitado que dissesse uma palavra que começasse com o mesmo som.

2.5. Síntese fonêmica: foram apresentadas à criança 21 palavras auditivamente separadas por sons e solicitado que esta dissesse a palavra formada.

2.6. Análise fonêmica: foram apresentadas à criança 21 palavras auditivamente e solicitado que dissesse os sons de cada letra das palavras apresentada.

2.7. Identificação de som inicial: foram apresentadas à criança 21 palavras auditivamente e solicitado que dissesse o som inicial da primeira letra de cada palavra apresentada.

3. Memória de trabalho: foram apresentadas à criança 24 pseudopalavras auditivamente e solicitado que repetisse como havia entendido.

4. Velocidade de acesso à informação fonológica: foram apresentadas à criança sete sequências intercalada de desenhos coloridos (carro, bola, pato, casa e chave) e solicitado que realizasse a nomeação rápida.

5. Atenção visual: foram apresentadas à criança 10 figuras coloridas e solicitado que esta identificasse entre duas palavras a que correspondia a cada figura.

6. Leitura de palavras e pseudopalavras: foram apresentadas à criança 40 palavras visualmente (20 palavras e 20 pseudopalavras) e solicitado que fosse realizada a leitura em voz alta.

7. Compreensão de frases a partir de figuras apresentadas: foram apresentadas à criança 20 frases incompletas com figuras ilustrativas e solicitado que esta observasse as figuras e completasse as frases.

A aplicação deste procedimento durou em média 50 minutos e foi realizado individualmente em apenas uma sessão durante o horário de aula das crianças, com a anuência e autorização da professora e da direção da escola em que foi realizado o estudo.

O material linguístico utilizado para a elaboração das provas descritas foi retirado de um banco de palavras de livros didáticos de $1^{\underline{a}}$ a $4^{\underline{a}}$ série utilizados na rede municipal de ensino de Marília-SP, sendo que as palavras utilizadas para elaboração das provas seguem as regras de decodificação do português brasileiro tanto para palavras como para pseudopalavras: a) regra de correspondência grafofonêmica independente do contexto e b) regra de correspondência grafofonêmica dependente do contexto ${ }^{14}$.

A caracterização do desempenho das 30 crianças deste estudo no Teste para identificação precoce dos problemas de leitura está descrita na Tabela 2.

As crianças que apresentaram valor mínimo, inferior a $51 \%$ ao valor máximo, em pelo menos quatro provas do teste para identificação precoce dos problemas de leitura em relação ao grupoclasse foram consideradas de risco para dislexia. Dentre as 30 crianças (100\%) submetidas ao procedimento de identificação dos sinais da dislexia, 15 crianças (50\%) apresentaram desempenho inferior. A partir deste achado, as crianças foram divididas em dois grupos:

- Grupo I (Gl): composto por 15 crianças submetidas ao programa de treinamento das habilidades fonológicas e correspondência grafemafonema, sendo $33 \%$ do gênero masculino e $18 \%$ do gênero feminino.

- Grupo II (GII): composto por 15 crianças não submetidas ao programa de treinamento das habilidades fonológicas e correspondência grafema-fonema, sendo $18 \%$ do gênero masculino e $33 \%$ do gênero feminino. 
Tabela 2 - Distribuição da média, desvio padrão, valor mínimo e valor máximo do desempenho obtido pelas crianças em cada prova do teste para identificação precoce dos problemas de leitura em situação de pré-testagem

\begin{tabular}{ccccc}
\hline Par de Variáveis & Média & Desvio padrão & Mínimo & Máximo \\
\hline CA & 19,63 & 3,18 & 12,00 & 23,00 \\
PR & 5,30 & 5,28 & 0,00 & 18,00 \\
IR & 11,90 & 5,96 & 0,00 & 20,00 \\
SS & 20,03 & 1,67 & 15,00 & 21,00 \\
PP & 16,87 & 3,66 & 7,00 & 22,00 \\
SF & 0,90 & 3,65 & 0,00 & 19,00 \\
AF & 2,27 & 2,95 & 0,00 & 13,00 \\
ISI & 4,80 & 7,17 & 0,00 & 21,00 \\
MT & 19,13 & 3,26 & 13,00 & 24,00 \\
AV & 9,37 & 1,19 & 6,00 & 10,00 \\
L & 20,30 & 15,30 & 0,00 & 40,00 \\
CF & 17,37 & 4,84 & 0,00 & 20,00 \\
\hline
\end{tabular}

Legenda: CA: conhecimento do alfabeto, PR: produção de rima, IR: identificação de rima, SS: segmentação silábica, PP: produção de palavras a partir do fonema dado, SF: síntese fonêmica, AF: análise fonêmica; ISI: identificação de som inicial, MT: memória de trabalho; AV: atenção visual, L: leitura, CF: compreensão de frases. Análise estatística: Teste dos Postos Sinalizados de Wilcoxon.

A partir da distribuição das crianças em dois grupos, foi realizada a aplicação do procedimento de intervenção individualmente. Assim como a avaliação utilizada anteriormente neste estudo, foi elaborada uma estratégia do programa de treinamento das habilidades fonológicas a partir da descrição da pesquisa original ${ }^{3}$.

O programa de treinamento das habilidades fonológicas e correspondência grafema-fonema utilizado neste estudo foi composto por 13 atividades trabalhadas em 18 sessões de 50 minutos de duração, realizado em duas sessões semanais na escola de origem das crianças. As atividades desenvolvidas no programa de treinamento estão descritas a seguir:

1. Identificação das letras e reconhecimento do alfabeto fonêmico: foram apresentados à criança os 23 grafemas para identificar o nome e o valor sonoro de cada letra.

2. Combinação de letra para formação de sílabas e formação de palavras: foi apresentado nas sessões iniciais um conjunto de quatro letras para a criança combinar (consoantevogal, consoante-consoante-vogal) compondo sílabas simples, sílabas complexas e posterior formação de palavras para a leitura. A partir da quarta sessão, o conjunto foi aumentado para 6 letras.

3. Identificação de figuras pelo nome das letras: foram apresentadas figuras para a criança identificar qual delas começava com a letra visualmente apresentada.
4. Identificação de figuras pelo som das letras: foram apresentadas figuras para a criança identificar qual delas iniciava com o som produzido pela pesquisadora.

5. Identificação de palavras dentro de uma frase: foram apresentadas sete frases afirmativas auditivamente para a criança dividir a frase em palavras, marcando-as por palmas.

6. Identificação e manipulação de sílabas na palavra: foram apresentadas duas palavras auditivamente para a identificação das sílabas iniciais e logo após, foi solicitada a manipulação de segmentação silábica para formação de novas palavras em posição inicial, medial e final. Em cada sessão foram fornecidas seis palavras.

7. Síntese fonêmica: foram apresentadas sete palavras auditivamente separadas por sons e solicitado à criança para reconhecer as palavras.

8. Rima: foram solicitadas para a criança palavras que terminassem com o mesmo som e que identificasse figuras que rimem em sua nomeação.

9. Identificação e discriminação de fonemas: foi apresentado auditivamente um fonema e solicitado à criança que mencionasse uma palavra que começasse com este som. Em seguida, foram apresentadas oralmente sete palavras e questionado se havia o fonema alvo naquela palavra. 
10. Segmentação de fonemas: foi apresentada uma palavra oralmente e solicitado à criança para falar todos os fonemas desta. Nesta fase do programa foram utilizadas fichas coloridas para auxiliá-la a segmentar os sons das palavras auditivamente apresentadas. Em cada sessão foram fornecidas sete palavras.

11. Subtração de fonemas: foram apresentadas à criança seis palavras oralmente para que retirasse o fonema final, e, em seguida, apresentadas seis palavras oralmente para que retirasse o fonema inicial.

13. Substituição de fonemas: foi apresentada oralmente à criança uma palavra e solicitado que retirasse o fonema inicial e o substituísse por outro, formando assim novas palavras. Em cada sessão foram fornecidas sete palavras.

13. Transposição de fonemas: foi solicitado à criança para falar as palavras em ordem inversa da palavra apresentada pela pesquisadora, formando, assim, novas palavras.

Depois do término do treinamento das habilidades fonológicas e correspondência grafemafonema, as crianças do GI e Gll foram submetidas novamente à aplicação do teste para a identificação precoce dos problemas de leitura.

Ressalta-se que, em atenção aos aspectos éticos em pesquisa com seres humanos, as crianças de risco para dislexia identificadas neste estudo e submetidas ao programa de treinamento, quando finalizado o tempo previsto para o treinamento, foram encaminhadas para diagnóstico interdisciplinar e tratamento fonoaudiológico no Centro de Estudos da Educação e Saúde - CEES/FFC/UNESP se em situação de pós-testagem não tivesse ocorrido a melhora das habilidades avaliadas na primeira testagem.

Este estudo foi aprovado pelo Comitê de Ética em Pesquisa da Faculdade de Filosofia e Ciências da Universidade Estadual Paulista - CEP/FFC/ UNESP sob o protocolo no 3348/2008.

Para a análise estatística foi utilizado o Teste de Friedman, com o intuito de verificar possíveis diferenças entre as variáveis do programa de treinamento das habilidades fonológicas e de correspondência letra-som. Outro método de análise estatística utilizado foi a aplicação do Teste dos Postos Sinalizados de Wilcoxon, com o objetivo de verificar possíveis diferenças entre os momentos de pré e pós-testagem, considerados na avaliação de cada grupo. O nível de significância adotado para a aplicação dos testes estatísticos foi de $5 \%$ $(0,050)$. A análise dos dados foi realizada utilizando - programa SPSS (Statistical Package for Social Sciences), em sua versão 13.0.

\section{RESULTADOS}

A Tabela 3 apresenta a distribuição da média, desvio padrão e valor de $p$ do desempenho obtido do GI e Gll no Teste para a identificação precoce dos problemas de leitura em situação de pré e póstestagem. Comparando os dois momentos de avaliação por meio do Teste dos Postos Sinalizados de Wilcoxon foi possível observar que as crianças do Gl submetidas ao programa de intervenção apresentaram diferença estatisticamente significante para as provas de conhecimento do alfabeto (CA), produção de rima (PR), identificação de rima (IR), segmentação silábica (SS), produção de palavra a partir do fonema dado (PP), síntese fonêmica (SF), análise fonêmica (AF), identificação do som inicial (ISI), memória de trabalho (MT), atenção visual (AV), leitura de palavras e não-palavras (L) e compreensão de frases a partir de figuras (CF), indicando melhora nas habilidades fonológicas e na leitura.

Entretanto, as crianças do Gll apresentaram diferença estatisticamente significante nas provas de produção de rima (PR), identificação de rima (IR) e leitura de palavras e não-palavras (L), demonstrando que o conteúdo oferecido pelo professor em sala de aula favoreceu a melhora do desempenho destas crianças nas provas descritas anteriormente (Tabela 3).

Em situação de pós-testagem, as crianças do GI e Gll obtiveram desempenho próximos em atividades como: conhecimento do alfabeto (CA), segmentação silábica (SS), memória de trabalho (MT), atenção visual (AV) e compreensão de frases a partir de figuras (CF).

Porém, nessa mesma situação, as crianças do Gl obtiveram resultados superiores às crianças do GII nas provas de conhecimento do alfabeto (CA), produção de rima (PR), identificação de rima (IR), produção de palavra a partir do fonema dado (PP), síntese fonêmica (SF), análise fonêmica ( $\mathrm{AF}$ ), identificação do som inicial (ISI), memória de trabalho (MT) e compreensão de frases a partir de figuras (CF), evidenciando a influência dessas habilidades como sendo importantes preditoras para a aquisição e desenvolvimento da leitura.

Conforme se observa na Tabela 4, as crianças do Gl em situação de pré-testagem apresentaram maior tempo de nomeação rápida de figuras no subteste para velocidade de acesso à informação fonológica do que as crianças do Gll, e que este tempo decresce na pós-testagem.

Apesar de não ter ocorrido diferença estatisticamente significante, este dado demonstra que o programa favoreceu a fusão rápida de estímulos em sucessão, exigida tanto em tarefas de nomeação rápida como em leitura. 
Tabela 3 - Distribuição da média, desvio padrão e valor de $p$ do desempenho obtido das crianças do GI e GIl em situação de pré e pós-testagem

\begin{tabular}{|c|c|c|c|c|c|c|c|}
\hline GI & Média & $\begin{array}{l}\text { Desvio } \\
\text { padrão }\end{array}$ & Valor de $p$ & GII & Média & $\begin{array}{l}\text { Desvio } \\
\text { padrão }\end{array}$ & Valor de $p$ \\
\hline CA_Pré & 18,33 & 3,33 & \multirow{2}{*}{$0,001^{*}$} & CA_Pré & 20,93 & 2,49 & \multirow{2}{*}{0,398} \\
\hline CA_Pós & 22,07 & 0,59 & & CA_Pós & 21,13 & 3,00 & \\
\hline PR_Pré & 2,93 & 4,01 & \multirow{2}{*}{$0,001^{*}$} & PR_Pré & 7,67 & 5,45 & \multirow{2}{*}{$0,009^{*}$} \\
\hline PR_Pós & 17,00 & 4,28 & & PR_Pós & 11,73 & 4,42 & \\
\hline IR_Pré & 10,07 & 6,45 & \multirow{2}{*}{$0,001^{*}$} & IR_Pré & 13,73 & 4,98 & \multirow{2}{*}{$0,022^{*}$} \\
\hline IR_Pós & 18,47 & 1,64 & & IR_Pós & 16,27 & 3,11 & \\
\hline SS_Pré & 19,40 & 2,13 & \multirow{2}{*}{$0,049^{*}$} & SS_Pré & 20,67 & 0,62 & \multirow{2}{*}{0,157} \\
\hline SS_Pós & 20,73 & 0,80 & & SS_Pós & 20,93 & 0,26 & \\
\hline PP_Pré & 15,13 & 3,85 & \multirow{2}{*}{$0,001^{*}$} & PP_Pré & 18,60 & 2,53 & \multirow{2}{*}{0,138} \\
\hline PP_Pós & 21,00 & 0,00 & & PP_Pós & 19,67 & 1,76 & \\
\hline SF_Pré & 0,07 & 0,26 & \multirow{2}{*}{$0,000^{*}$} & SF_Pré & 1,73 & 5,11 & \multirow{2}{*}{0,123} \\
\hline SF_Pós & 18,60 & 5,04 & & SF_Pós & 4,33 & 7,05 & \\
\hline AF_Pré & 1,07 & 1,62 & \multirow{2}{*}{$0,001^{*}$} & AF_Pré & 3,47 & 3,50 & \multirow{2}{*}{0,187} \\
\hline AF_Pós & 18,40 & 4,58 & & AF_Pós & 7,47 & 8,63 & \\
\hline ISI_Pré & 0,80 & 1,74 & \multirow{2}{*}{$0,000^{*}$} & ISI_Pré & 8,80 & 8,33 & \multirow{2}{*}{0,813} \\
\hline ISI_Pós & 20,67 & 1,05 & & ISI_Pós & 7,40 & 7,50 & \\
\hline MT_Pré & 18,20 & 3,23 & \multirow{2}{*}{$0,003^{*}$} & MT_Pré & 20,07 & 3,11 & \multirow{2}{*}{0,582} \\
\hline MT_Pós & 21,87 & 1,55 & & MT_Pós & 20,73 & 2,69 & \\
\hline VIF_Pré & 41,20 & 11,65 & \multirow{2}{*}{0,232} & VIF_Pré & 35,27 & 5,74 & \multirow{2}{*}{0,421} \\
\hline VIF_Pós & 39,60 & 9,83 & & VIF_Pós & 32,87 & 6,41 & \\
\hline AV_Pré & 8,80 & 1,47 & \multirow{2}{*}{$0,017^{*}$} & AV_Pré & 9,93 & 0,26 & \multirow{2}{*}{0,317} \\
\hline AV_Pós & 9,80 & 0,41 & & AV_Pós & 10,00 & 0,00 & \\
\hline L_Pré & 11,20 & 12,73 & \multirow{2}{*}{$0,001^{*}$} & L_Pré & 29,40 & 12,06 & \multirow{2}{*}{$0,019^{*}$} \\
\hline L_Pós & 26,67 & 8,73 & & L_Pós & 34,53 & 6,83 & \\
\hline CF_Pré & 15,80 & 6,32 & \multirow{2}{*}{$0,005^{\star}$} & CF_Pré & 18,93 & 1,83 & \multirow{2}{*}{0,931} \\
\hline CF_Pós & 20,00 & 0,00 & & CF_Pós & 19,27 & 1,34 & \\
\hline
\end{tabular}

Legenda: CA-Conhecimento do alfabeto, PR-Produção de rima, IR-Identificação de rima, SS-Segmentação silábica, PP-Produção de palavra a partir do fonema dado, SF-Síntese fonêmica, AF-Análise fonêmica, ISI-Identificação do som inicial, MT-Memória de trabalho, VIF-Velocidade de acesso à informação fonológica, AV-Atenção visual, L-Leitura de palavras e não-palavras, CF-Compreensão de frases a partir de figuras, (GI)-crianças submetidas à intervenção, (GII)-crianças não submetidas à intervenção. Análise estatística: Teste dos Postos Sinalizados de Wilcoxon.

Tabela 4 - Distribuição da média, desvio padrão, valor mínimo, valor máximo e valor de $p$ do desempenho das crianças do GI e Gll em situação de pré e pós-testagem no subteste de nomeação rápida de figuras (NR)

\begin{tabular}{ccccccc}
\hline & $\begin{array}{c}\text { Par de } \\
\text { Variáveis }\end{array}$ & Média & $\begin{array}{c}\text { Desvio } \\
\text { padrão }\end{array}$ & Mínimo & Máximo & \multirow{2}{*}{ Valor de $\mathbf{p}$} \\
\hline \multirow{2}{*}{ GI } & NR pré & 41,20 & 11,65 & 29,00 & 71,00 & \multirow{2}{*}{0,232} \\
& NR pós & 39,60 & 9,83 & 30,00 & 66,00 & \\
\hline \multirow{2}{*}{ GII } & NR pré & 35,27 & 5,74 & 28,00 & 50,00 & \multirow{2}{*}{0,421} \\
& NR pós & 32,87 & 6,41 & 23,00 & 45,00 & \\
\hline
\end{tabular}


Dentre as 15 crianças (100\%) submetidas ao programa de treinamento de habilidades fonológicas e correspondência grafema-fonema, apenas uma criança $(3 \%)$ continuou apresentando valor mínimo inferior a $51 \%$ em quatro provas do teste para identificação precoce dos problemas de leitura em relação ao grupo-classe em situação de póstestagem. Esta criança foi encaminhada para avaliação interdisciplinar composta por avaliação neurológica e neuropsicológica no Centro de Estudos da Educação e Saúde - CEES/FFC/UNESP em que foi confirmado o quadro de dislexia do desenvolvimento. Após o diagnóstico interdisciplinar, a menor permaneceu no CEES para intervenção fonoaudiológica semanal.

\section{DISCUSSÃO}

Os resultados revelaram que das 15 crianças consideradas de risco para a dislexia após serem submetidas ao programa de treinamento fonológico associado à correspondência letra-som, 14 crianças melhoraram o desempenho em estratégias de percepção, discriminação, armazenamento e recuperação de informação fonêmica, conforme descrito em estudos internacionais ${ }^{3,11,15,16}$.

Em decorrência disso, essas crianças apresentaram melhora nas habilidades cognitivo-linguísticas, pois ao melhorar o desempenho em percepção, discriminação e tempo de armazenamento da informação linguística, ocorre a melhora no uso de habilidades de leitura e compreensão, conforme descrito na literatura ${ }^{3}$.

A ênfase na identificação precoce nas crianças de risco para a dislexia ocorreu devido ao aumento de evidências na literatura ${ }^{6,7,13}$ de que as habilidades cognitivas e linguísticas quando trabalhadas precocemente com essas crianças no período préescolar influenciam na aquisição do princípio alfabético do sistema de escrita.

O programa de treinamento adaptado neste estudo enfocou a atenção, discriminação e memória auditiva e visual de forma direta em todas as sessões, o que favoreceu as crianças submetidas ao programa melhor e maior tempo de atenção à base fonológica do sistema de escrita da Língua Portuguesa. Por isso, as crianças com sinais da dislexia apresentaram melhora nas habilidades fonológicas, apontando para a necessidade de uma reflexão sobre a metodologia de alfabetização utilizada em situação de sala de aula, pois as habilidades de atenção, discriminação e percepção dos sons e letras trabalhadas neste programa proporcionaram aumento na sensibilidade e percepção fonológica, o que se refletiu também na melhora do tempo de realização da leitura. Estes mesmos achados foram descritos por autores internacionais em seus estudos ${ }^{3,11,15-18}$.

O desempenho demonstrado pelas crianças depois de serem submetidas ao programa de treinamento, em relação às habilidades cognitivolinguísticas, apresentou resultado significativo, evidenciando que a instrução explícita da consciência fonológica combinada à instrução da correspondência grafema-fonema acelera a aquisição da leitura. Esses achados corroboram um estudo descrito na literatura ${ }^{19-21}$, pois o ensino da base alfabética de uma língua auxilia o desenvolvimento da consciência fonológica, que, por sua vez, facilita a aquisição e desenvolvimento da leitura.

Entretanto, destaca-se que depois do programa de treinamento fonológico e de correspondência grafema-fonema realizado com as 15 crianças consideradas de risco para a dislexia, apenas uma criança não apresentou melhora no desempenho em situação de pós-testagem. Desse modo, 14 crianças apresentavam apenas falha no processo de alfabetização e não o quadro de dislexia. Esses achados remetem à reflexão de que os sinais descritos na literatura nacionais e internacionais $2,11,15,22$ sofrem interferência direta da metodologia de ensino, o que justifica o fato de os fonoaudiólogos juntamente com os professores utilizarem programas de intervenção com base fonológica e na relação letra-som para identificar e intervir precocemente nos sinais da dislexia e, consequentemente, diminuir o número de encaminhamentos desnecessários para a realização de diagnóstico fonoaudiológico. Essa prática corrobora estudos descritos na literatura internacional ${ }^{16-18,23-27 .}$

Assim, os achados deste estudo apresentam o treinamento da habilidade fonológica e correspondência grafema-fonema em crianças de $1^{\underline{a}}$ série com risco para dislexia como um instrumento de auxílio ao diagnóstico de uma condição determinada genética e neurologicamente como a dislexia, uma vez que conforme descrição na literatura internacional ${ }^{3,5,6,12,13}$, o fracasso da criança de risco a este tipo de treinamento é um critério diagnóstico para a dislexia. Esses achados apontam para o fato de que quanto menor for a instrução na base fonológica e da relação letra-som para a aprendizagem da leitura, maior será o risco de os fonoaudiólogos identificarem erroneamente uma criança como sendo disléxica.

\section{CONCLUSÃO}

A realização do programa de treinamento das habilidades fonológicas e correspondência grafema-fonema foi eficaz para a identificação das crianças com sinais da dislexia, o que pode ser 
comprovado pela melhora das habilidades fonológicas e de leitura em situação de pós-testagem em relação à pré-testagem.

Os dados referentes ao teste de identificação precoce nos problemas de leitura em situações pré e pós-testagem evidenciaram a importância da realização de uma avaliação que enfoque as habilidades fonológicas e a relação letra-som no âmbito clínico e educacional, visto que isso auxiliaria as crianças em situação de alfabetização a desenvolverem habilidades cognitivo-linguísticas necessárias para a aprendizagem da base alfabética do sistema de escrita do português brasileiro. Somente por meio desta instrução formal, seria possível garantir que as crianças que não apresentassem aprendizagem do mecanismo de relação letrasom fossem eletivas para o diagnóstico da dislexia e a distinção entre o mau leitor e o leitor disléxico poderia ser finalmente reconhecida entre os profissionais que atuam no diagnóstico e intervenção dos problemas de aprendizagem.

\section{AGRADECIMENTOS}

Ao CNPq pela concessão da bolsa de produtividade em pesquisa para a segunda autora.

\begin{abstract}
Purpose: to check the efficacy of the phonological training and grapheme-phoneme correspondence program in first-grade children under dyslexia risk. Method: thirty municipal public study students of both genders from $1^{\text {st }}$ grade took part, ranging from 6 to 7 year old. In this study we used the Brazilian adaptation of the research on phonological abilities training and grapheme-phoneme correspondence composed of pre-testing, training, and post-testing. In the pre and post-testing situation, all children were submitted to the test for early identification of reading problems and those who presented less than $51 \%$ of the tasks of the test were divided into Group I (GI): consisted of 15 children who were submitted to the training program; and Group II (GII): consisted of 15 children who were not submitted to the training program. Results: the results of this study demonstrated statistically significant differences, indicating that from 15 children who were submitted to the program, just 1 child did not answer the proposed intervention, being submitted to interdisciplinary evaluation and confirmed the dyslexia diagnosis. Conclusion: the phonological ability training and grapheme-phoneme correspondence program were effective for identifying children with dyslexia symptoms, proven by the improvement of the phonological abilities and of reading in the post-testing situation compared to pre-testing, indicating that when the formal instruction concerning the alphabetical principle of the Portuguese language is provided, children who do not show dyslexia stop demonstrating such manifestations as an answer to the formal instruction of the alphabetical principle.
\end{abstract}

KEYWORDS: Dyslexia; Evaluation; Early Intervention (Education); Reading; Child

\section{REFERÊNCIAS}

1. Lyon GR, Shaywitz SE, Shaywitz BA. Defining dyslexia, comorbidity, teacher's knowledge of language and reading. Ann Dyslexia. 2003; 53(1):1-14.

2. ASHA: American Speech and Hearing Association [homepage na internet]. Rockville: American Speech-Language-Hearing Association; c1997-2008. Language-based learning disabilities. [acesso em 29 Ago 2003]. Disponível em: http:// www.asha.org.

3. Schneider W, Roth E, Ennemoser M. Training phonological skills and letter knowledge in children at risk for dyslexia: a comparison of three kindergarten intervention programs. J Educ Psychol. 2000; 92(2):284-95.

4. Agnew JA, Dorn C, Eden GF. Effect of intensive training on auditory processing and reading skills. Brain Lang. 2004; 88(1):21-5.

5. Elbro C, Petersen DK. Long-term effects of phoneme awareness and setter sound training: an intervention study with children at risk for dyslexia. J Educ Psychol. 2004; 96(4):660-70.

6. Gijsel MAR, Bosman AMT, Verhoeven L. Kindergaten risk factors, cognitive factors, and teacher judgments as predictors of early reading in dutch. J Learn Disabil. 2006; 39(6):558-71. 
7. Molfese VJ, Modglin AA, Beswick JL, Neamon JD, Berg SA, Berg CJ, et al. Letter knowledge, phonological processing, and print knowledge: skill development in non-reading preschool children. J Learn Disabil. 2006; 39(4):296-305.

8. Schatschneider C, Fletcher JM, Francis, DJ, Carlson, CD, Foorman BR. Kindergarten prediction of reading skills: a longitudinal comparative analysis. J Educ Psychol. 2004; 96(2):265-82.

9. Jong PF. Phonological awareness and the use of phonological similarity in letter-sound learning. $J$ Exp Child Psychol. 2007; 98:131-52.

10. Share DL. Knowing letter names and learning letter sounds: a causal connection. J Exp Child Psychol. 2004; 88:213-33.

11. López-Escribano C, Beltrán JA. Early predictors of reading in three groups of native Spanish speakers: Spaniards, Gypsies, and Latin Americans. Span J Psychol. 2009; 12:84-95.

12. Vaughn $S$, Linan-Thompson $S$, Mathes PG, Cirino PT, Carlson CD, Pollard-Durodola SD, et al. Effectiveness of spanish intervention for firstgrade English language learners at risk for reading difficulties. J Learn Disabil. 2006; 39(1):56-73.

13. Landry SH, Swank PR, Smith KE, Assel MA, Gunnewig, SB. Enhancing early literacy skills for preschool children; bringing professional development model to scale. J Learn Disabil. 2006; 39(4):306-24.

14. Scliar-Cabral L. Princípios do sistema alfabético do português do Brasil. São Paulo: Contexto; 2003.

15. Etchepareborda MC. La intevencíón en los transtornos disléxicos: entrenamiento de la conciencia fonológica. Rev Neurol. 2003; 36:13-9.

16. Harn BA, Linan-Thompson S, Roberts G. Intensifying instruction: does additional instructional time make a difference for the most at-risk first graders?. J Learn Disabil. 2008; 41(2):115-25.

17. Simmons DC, Coyne MD, McDonagh S, Harn BA, Kame'enui EJ. Indexing response to intervention: a longitudinal study of reading risk from Kindergarten through third grade. J Learn Disabil. 2008; 41(2):158-73.

18. López-Escribano C. Contribuciones de la neurociencia al diagnóstico y tratamiento educativo de la dislexia del desarrollo. Rev Neurol. 2007; 44(3):173-80.

19. Stackhouse J. Fala, ortografia e leitura: quem está em risco e por quê? In: Snowling $M$, Stackhouse J. Dislexia, fala e linguagem: um manual do profissional. Porto Alegre: Artmed; 2004. p. 280-324.

20. Cunha VLO, Capellini SA. Desempenho de escolares de $1^{\text {a }}$. à $4^{a}$. Série do ensino fundamental nas provas de habilidades metafonológicas e de leitura-PROHMELE. Rev Soc Bras Fonoaudiol. 2009; 14(1):56-68.

21. Smythe I, Everatt J, Al-Menaye N, He X, Capellini SA, Gyarmathy E, et al. Predictors of world-level literacy amongst grade 3 children in five diverse languages. Dyslexia. 2008; 14(3):170-87.

22. Capellini SA. Distúrbios de aprendizagem versus dislexia. In: Ferreira LP, Befi-Lopes DM, Limongi SCO, organizadores. Tratado de fonoaudiologia. São Paulo: Editora Roca; 2004. p. 862-76.

23. Simmons DC, Kame'enui EJ, Harn B, Coyne MD, Stoolmiller M, Santoro LE, et al. Attributes of effective and efficient kindergarten reading intervention: an examination of instructional time and design of instruction specificity. J Learn Disabil. 2007; 40:331-47.

24. Simos PG, Sarkari S, Billingsley RL, Fletcher JM, Denton C, Papanicolaou AC. Altering the brain circuits for reading through intervention: a magnetic source imaging study. Neuropsychol. 2007; 21(4):485-6.

25. Broom YM, Doctor EA. Developmental phonological dyslexia: a case study of the efficacy of a remediation programme. Cognit Neuropsychol. 1995; 12(7):725-66.

26. Calhoon MB. Effects of a peer-mediated phonological skill and reading comprehension program on reading skill acquisition for middle school students with reading disabilities. J Learn Disabil. 2005; 38(5):424-33.

27. Fletcher JM, Coulter WA, Reschly DJ, Vaughn S. Alternative approaches to the definition and identification of learning disabilities: some questions and answers. An Dys. 2004; 54(2):304-31.

DOI: 10.1590/S1516-18462010005000074

RECEBIDO EM: 18/12/2009

ACEITO EM: 04/05/2010

Endereço para correspondência:

Simone Aparecida Capellini

Av. Hygino Muzzi Filho, 737

Marília - SP

CEP: $17525-900$

E-mail: sacap@uol.com.br 